\title{
Recent developments in ferroelectric nanostructures and multilayers
}

\author{
S. P. Alpay · V. Nagarajan · G. A. Rossetti Jr.
}

Published online: 11 August 2009

(C) Springer Science+Business Media, LLC 2009

The property of ferroelectricity was first reported in 1921 in Rochelle salt (sodium potassium tartrate). Once considered a rare phenomenon limited to a comparatively small class of crystals, the discovery of ferroelectricity in the binary oxide compound barium titanate $\left(\mathrm{BaTiO}_{3}\right)$ in the mid-1940s paved the way for rapid advances in the search of new materials. Since that time, a great many other ferroelectric compounds and solid solutions have been found and there has been broad interest in ferroelectric materials that has continued essentially uninterrupted until the present time. Ferroelectric materials remain subjects of intensive investigation today for three principal reasons. First, the unique dielectric, pyroelectric, piezoelectric, and electro-optic properties exhibited by ferroelectric crystals, ceramics, composites, and thin films can be exploited in great many devices of commercial importance. Second, apart from their many technological applications, ferroelectric materials as a class exhibit a great diversity of phase transitions that make them ideal objects for scientific investigations into the origins and mechanisms of a wide range of structural transformation phenomena. Finally,

S. P. Alpay $(\varangle) \cdot$ G. A. Rossetti Jr.

Materials Science and Engineering Program, Departments

of Chemical, Materials, and Biomolecular Engineering,

University of Connecticut, Storrs, CT, USA

e-mail: p.alpay@ims.uconn.edu

G. A. Rossetti Jr.

e-mail: rossetti@ims.uconn.edu

V. Nagarajan

School of Materials Science and Engineering, University of New

South Wales, Sydney, NSW, Australia

e-mail: nagarajan@unsw.edu.au advances in thin-film deposition and nanoscale fabrication techniques made over the past two decades have created new possibilities for the integration of these materials into the ever expanding array of microelectronic devices.

Ferroelectrics form a sub-group of functional (or smart) materials whose physical properties are sensitive to changes in external conditions such as temperature, pressure, and electric fields. Below some critical temperature $T_{\mathrm{C}}$, the dielectric displacement (electric polarization) spontaneously assumes non-zero values in the absence of any externally applied force. The transition between non-ferroelectric and ferroelectric phases is accompanied by a loss of symmetry, characterized by double-well minima below the transition temperature, resulting in a switchable polarization that is accompanied by a hysteresis in the electric field-dielectric displacement response. As a class of materials, ferroelectrics also exhibit unusually large and nonlinear generalized susceptibilities; their dielectric, piezoelectric, elastic, and other properties display critical behavior near the ferroelectric phase transformation temperature. Ferroelectrics may be regarded as high energydensity materials as well that can be configured to store, release or interconvert electrical and mechanical energy in a well-controlled manner. Their exceptionally large piezoelectric compliances, pyroelectric coefficients, and dielectric susceptibilities can be exploited in a variety of microelectronic devices. Important examples of these include piezoelectric sensors and actuators, pyroelectric thermal imaging devices, high-dielectric constant capacitors, electro-optic light valves, and thin-film memories. Because of their strongly non-linear dielectric response ferroelectrics can be utilized in applications such as frequency-agile phase shifters and filters in wireless telecommunications systems.

Because the properties of ferroelectric materials that are exploited in technological applications are intimately 
connected with lattice instabilities, the physics of ferroelectric phase transformations also continues to be an active area of research. For example, the broad array of ferroelectric, antiferroelectric, ferrodistortive, and antiferrodistorive transformations observed in oxide materials are somewhat unique among those found in functional materials in that they do not involve an intrinsic change in electronic or magnetic structure. The theory of ferroelectricity has thus proven to be a fertile ground where quasistatic Landau-type thermodynamic models, dynamic phase field approaches, and ab initio computations have all been employed. In recent years, these methods have been successfully applied to understand the role of internal stresses in ferroelectric films, interlayer coupling in multilayer ferroelectrics, reduced size scaling, surface-induced strain effects, and domain morphologies and polarization variations in ferroelectric films and nanostructures.

We note that the field of multiferroics has also seen a resurgence of scientific and technological interest. Multiferroics possess more than one ferroic order parameter (polarization, magnetization, and/or self-strain). A particularly active area of research currently focuses on magnetoelectric materials that have both strong spontaneous electric polarization and magnetization and a number of perovskite-based compounds with interesting multiferroic property responses have been reported. Research on multiferroics has also involved concurrent efforts in the area of artificial magnetoelectrics that consist of nano- or mesoscale composites comprising ferromagnetic and ferroelectric materials wherein magnetoelectric coupling is established through internal strain, and/or electro- and magnetostatic interactions.

In this Special Issue of the Journal of Materials Science we present a collection of experimental and theoretical articles that concentrate on the most recent and exciting developments in ferroelectric materials and phenomena. A broad range of topics is covered including nanoscale synthesis and characterization of ferroelectric materials and devices, physics of ferroelectrics in the nanoscale, multiferroics and multilayer ferroelectric heterostructures, defects and domain microstructure, electromechanical (piezoelectric), pyroelectric and electrocaloric properties, and ferroelectrics and multiferroic materials for tunable devices. This special issue contains several review papers but is largely devoted to articles concentrating on cuttingedge areas in ferroelectrics research.

The special issue starts off with three detailed general review articles on the developments in the field of strain engineering in ferroelectric thin films [1], lead-free piezoelectrics [2], and photochemistry of ferroelectrics [3]. Yan et al. [4] provide an overview of magnetoelectric nanocomposites made up of perovskite ferroelectrics and spinel ferromagnetics. Magnetoelectrics are treated at length; topics include defects in $\mathrm{BiFeO}_{3}$, crystal chemistry of rare-earth doped $\mathrm{BiFeO}_{3}$, and strain-induced artificial magnetoelectricity in $\mathrm{Pb}\left(\mathrm{Zr}_{0.53} \mathrm{Ti}_{0.47}\right) \mathrm{O}_{3}-\mathrm{Pb}\left(\mathrm{Fe}_{0.66} \mathrm{~W}_{0.33}\right) \mathrm{O}_{3}$ [5-10].

The aggressive "scaling down" of ferroelectric materials for microelectronic device applications have brought with it issues related to the finite size effect in ferroelectrics. A general theoretical approach concerning the properties of ferroelectrics in the nanoscale is framed by Eliseev and Morovoska [11]. The synthesis of ferroelectric nanocubes and epitaxial nanostructures are discussed by Wada et al. [12] and Han et al. [13]. Piezoresponse Force Microscopy (PFM) has been proven to be an invaluable tool to understand the properties of ferroelectrics in the nanoscale. This special issue contains several contributions from leading groups that specialize in this technique [5, 14-17] and its application to polarization switching [14], imaging ferroelectric and ferroelastic domains [16] as well as new high-speed methods that allow 20-nm spatial and 10-ns temporal resolution [15].

Besides the analysis of Eliseev and Morovoska [11], we include in this special issue on ferroelectrics excellent theoretical contributions that employ a variety of analytical and computational tools. To understand and to describe ferroelastic domains in ferroelectrics, Liu and $\mathrm{Li}$ use an equivalent inclusion model coupled with elastic compatibility relations [18]. Wang provides a detailed account of the application of phase-field approaches to the study of ferroelectric phase transformations and domain mechanisms [19]. First-principles calculations are carried out to predict hypertoroidal electric moments in ferroelectric nanostructures [20] and to describe the antiferroelectric behavior of $\mathrm{PbZrO}_{3}$ [21]. The effect of external stresses on the electromechanical properties is treated using a microscopic model [22]. Zhang et al. [23] determine electrocaloric and pyroelectric properties of bulk and thin film $\mathrm{PbTiO}_{3}, \mathrm{BaTiO}_{3}$, and $\mathrm{SrTiO}_{3}$ via a Landau-Devonshire type thermodynamic formalism.

In ferroelectric films and multilayer heterostructures, the interfaces between the substrate and the film, or between the electrode and the film, become critically important for technological applications. In addition to the electronic mismatch due to differences in the band structure, the misfit strain due to lattice parameter differences may result in defect microstructures that can alter ferroelectric properties. $\mathrm{Li}$ and Chen [24] provide a general overview of interface effects in terms of the defect morphology in epitaxial ferroelectric films and Jackson and Jones [25] discuss the role of defects on the dielectric tunability of BST. Arredondo et al. [26] investigate compositional variations across interfaces in epitaxial ferroelectric 
heterostructures using transmission electron microscopy (TEM) and spectroscopy. Elastic domains in bulk and thin-film ferroelectrics are analyzed in contributions from Catalan et al. [27], Suleimanov et al. [28], and Morioka et al. [29].

Recent developments in synthesis and processing of ferroelectric films are covered in four articles [30-33]. These include laser transfer processing [30], UV-photon irradiation [31], and orientation-controlled MOCVD on patterned buffer layers [32]. While not completely related to ferroelectrics, we also include a article on the deposition of metal-insulator transition in $\mathrm{VO}_{2}$; a material that may have the potential to act as a "smart" electrode in ferroelectric devices [33].

Another topic of great interest in recent years is ferroelectric multilayers and graded heterostructures which display many unique properties that are not observed in monolithic ferroelectric materials. These include an off-set in the polarization hysteresis loop along the polarization axis and temperature-insensitive property coefficients. While still being debated, the origins of these observations are believed to stem from elastic, electrostatic, and electromechanical interactions between the ferroelectric layers. The special issue contains four experimental and theoretical contributions that discuss ferroelectric multilayers and compositionally graded ferroelectrics. Misirlioglu et al. [34] employ a Monte Carlo simulation taking into account long-range dipolar interactions in their analysis of ferroelectric and antiferroelectric multilayers. Weiss et al. [35] consider electrostatic and strain coupling in compositionally graded BST and discuss the potential of such materials systems in frequency agile tunable devices. The structural and electrical properties of heterolayered PZT films with different stacking sequence and layer thicknesses are analyzed by Kartawidjaja et al. [36]. Using the theory of dense domain structures and elastic coupling in the continuum limit, Mahjoub et al. [37] show that the ferroelastic domain population in multilayered tetragonal-rhombohedral PZT multilayers may differ greatly from single-layer tetragonal PZT.

This special issue of the Journal of Materials Science also contains an overview on factors affecting the performance of piezoelectric actuators [38] and a study on the piezoelectric behavior of lead-free $\mathrm{Bi}_{1 / 2} \mathrm{Na}_{1 / 2} \mathrm{TiO}_{3}-\mathrm{K}_{1 / 2}$ $\mathrm{Na}_{1 / 2} \mathrm{NbO}_{3}$-based ceramics [39]. Recent developments in relaxor ferroelectrics are included in contributions from Wu et al. [40] and Wongmaneerung et al. [41].

Acknowledgements The guest editors would like to thank the Editor-in-Chief Professor C. B. Carter (University of Connecticut), the Associate Editor Professor M. Aindow (University of Connecticut) in charge of this special issue, the Journal of Materials Science staff, and the Senior Production Editor Mr. R. Maged at Springer for all their efforts in the publication of this special issue.

\section{References}

1. Janolin P-E. J Mater Sci. doi:10.1007/s10853-009-3553-1

2. Panda PK. J Mater Sci. doi:10.1007/s10853-009-3643-0

3. Tiwari D, Dunn S. J Mater Sci. doi:10.1007/s10853-009-3472-1

4. Yan L, Yang Y, Wang Z, Xing Z, Li J, Viehland D. J Mater Sci. doi:10.1007/s10853-009-3679-1

5. Maksymovych P, Balke N, Jesse S, Huijben M, Ramesh R, Baddorf AP, Kalinin SV. J Mater Sci. doi:10.1007/s10853009-3697-z

6. Karimi S, Reaney IM, Han Y, Pokorny J, Sterianou I. J Mater Sci. doi:10.1007/s10853-009-3545-1

7. Kumar A, Katiyar RS, Premnath RN, Rinaldi C, Scott JF. J Mater Sci. doi:10.1007/s10853-009-3503-y

8. Zhang N, Srinivasan G, Balbashov AM. J Mater Sci. doi: 10.1007/s10853-009-3455-2

9. Ortega N, Kumar A, Katiyar RS, Rinaldi C. J Mater Sci. doi: 10.1007/s10853-009-3635-0

10. Zhang JX, Dai JY, Lu W, Chan HLW. J Mater Sci. doi: 10.1007/s10853-009-3512-x

11. Eliseev EA, Morozovska AN. J Mater Sci. doi:10.1007/s10853009-3473-0

12. Wada S, Nozawa A, Ohno M, Kakemoto H, Tsurumi T, Kameshima Y, Ohba Y. J Mater Sci. doi:10.1007/s10853-009-3705-3

13. Han H, Lee K, Lee W, Alexe M, Hesse D, Baik S. J Mater Sci. doi:10.1007/s10853-009-3528-2

14. Gruverman A. J Mater Sci. doi:10.1007/s10853-009-3623-4

15. Polomoff NA, Premnath RN, Bosse JL, Huey BD. J Mater Sci. doi:10.1007/s10853-009-3699-x

16. McGilly L, Byrne D, Harnagea C, Schilling A, Gregg JM. J Mater Sci. doi:10.1007/s10853-009-3626-1

17. Nonnenmann SS, Spanier JE. J Mater Sci. doi:10.1007/ s10853-009-3680-8

18. Liu YY, Li JY. J Mater Sci. doi:10.1007/s10853-009-3536-2

19. Wang YU. J Mater Sci. doi:10.1007/s10853-009-3663-9

20. Prosandeev S. Bellaiche L. J Mater Sci. doi:10.1007/ s10853-009-3460-5

21. Pilania G, Tan DQ, Cao Y, Venkataramani VS, Chen Q, Ramprasad R. J Mater Sci. doi:10.1007/s10853-009-3465-0

22. Cao H-X, Lo VC, Li Z-Y. J Mater Sci. doi:10.1007/s10853009-3537-1

23. Zhang J, Heitmann AA, Alpay SP, Rossetti GA. J Mater Sci. doi: 10.1007/s10853-009-3559-8

24. Lin Y, Chen CL. J Mater Sci. doi:10.1007/s10853-009-3664-8

25. Jackson TJ, Jones IP. J Mater Sci. doi:10.1007/s10853009-3666-6

26. Arredondo M, Saunders M, Petraru A, Kohlstedt H, Vrejoiu I, Alexe M, Hesse D, Browning ND, Munroe P, Nagarajan V. J Mater Sci. doi:10.1007/s10853-009-3548-y

27. Catalan G, Lukyanchuk I, Schilling A, Gregg JM, Scott JF. J Mater Sci. doi:10.1007/s10853-009-3554-0

28. Suleimanov N, Milner A, Zon I, Roytburd A, Lubomirsky I. J Mater Sci. doi:10.1007/s10853-009-3642-1

29. Morioka H, Saito K, Yokoyama S, Oikawa T, Kurosawa T, Funakubo H. J Mater Sci. doi:10.1007/s10853-009-3606-5

30. James C, Chakraborty $T$, Brown A, Comyn T, Dorey R, Harrington J, Laister J, Miles RE, Puchmark C, Xu B, Xiong W, Zhang Q, Milne SJ. J Mater Sci. doi:10.1007/s10853-009-3609-2

31. Cole MW, Podpirka A, Ramanathan S. J Mater Sci. doi: 10.1007/s10853-009-3538-0

32. Nishida K, Yamamoto T, Osada M, Sakata O, Kimura S, Saito K, Nishide M, Katoda T, Yokoyama S, Funakubo H. J Mater Sci. doi:10.1007/s10853-009-3683-5

33. Gopalakrishnan G, Ruzmetov D, Ramanathan S. J Mater Sci. doi: 10.1007/s10853-009-3442-7 
34. Misirlioglu IB, Pintilie L, Alexe M, Hesse D. J Mater Sci. doi: 10.1007/s10853-009-3451-6

35. Weiss CV, Okatan MB, Alpay SP, Cole MW, Ngo E, Toonen RC. J Mater Sci. doi:10.1007/s10853-009-3514-8

36. Kartawidjaja FC, Sim CH, Wang J. J Mater Sci. doi: 10.1007/s10853-009-3569-6

37. Mahjoub R, Anbusathaiah V, Nagarajan V. J Mater Sci. doi: $10.1007 / \mathrm{s} 10853-009-3647-9$
38. Li T, Chen YH, Ma J. J Mater Sci. doi:10.1007/s10853009-3567-8

39. Xiao DQ, Wu JG, Wu L, Zhu JG, Yu P, Lin DM, Liao YW, Sun Y. J Mater Sci. doi:10.1007/s10853-009-3543-3

40. Wu L, Wang X, Wang JH, Guo R, Bhalla AS. J Mater Sci. doi: 10.1007/s10853-009-3686-2

41. Wongmaneerung R, Yimnirun R, Ananta S. J Mater Sci. doi: 10.1007/s10853-009-3621-6 